\title{
Modeling smart grids using closed loop supply chain concepts
}

\author{
Nathalie Saker", Laoucine Kerbache
}

HEC Paris, Doha, Qatar

\begin{abstract}
The traditional electrical power grid used to be one-directional power flows (flowing from producers to consumers). Nowadays, the consumers become more active and they participate in electricity generation process through Demand Response (DR) actions applied on their electrical loads. As a result, power flows become more and more multidirectional and production units more decentralized. Electrical grid architectures must be developed in parallel in order to meet these increasing changes that lead to what is known to be Smart Grids. In this paper, we first present a literature review about the smart grid architectures and the various associated models. Then, we propose a closed loop supply chain approach to model and optimize configurations and operations of smart grid systems. Within this context, a whole section is devoted to the development of a mixed nonlinear programming model to optimize the architecture of given smart grid systems. For illustrative purposes, using a specific smart grid system and appropriate data, we model the problem and solve it for the optimal architecture.
\end{abstract}

Keywords: Smart grid architecture, closed loop supply chain, mathematical model and optimization

\section{Introduction}

The power system landscape is being transformed from a centralized and one-directional power flows toward a Smart Grid with a decentralized structure having multidirectional power flows; the reason for that is the increasingly involving of end use consumers in the electricity generation process through having their consumption controlled via Demand Response (or DR) actions.

When talking about smart grids, there are two fields for addressing them: (i) the smart electrical power network like the one described in [1], and (ii) the smart telecommunication network like the one presented in [2]. The focus in this study will be on the smart electrical power network.

The Smart Grid consists principally of two ways direction flows (the first direction flows is from the producer to the consumer and the other one is from the consumer back to the electrical grid). The power flow from consumers back to the grid could be generated from two sources: (i) from the renewable energy generation units installed in the accommodations' levels (or distribution grid levels) or (ii) from Demand Response (or DR) actions that consist of controlling electrical loads so they can be interrupted during a certain period, and so the controlled power (that was supposed to be consumed) could be reinjected into the electrical system, and that will be the focus of our study; a way to represent this two-way directional power flows is to use the classical supply chain structure where a plant can manufacture new products and remanufacture returned ones; in our case, the manufactured new products and the returned ones are simply the electrical power.

The linkage between smart grids and the closed loop supply chain was only addressed by very few authors like [3]. However, they did not develop any mathematical model of smart grid systems. Therefore, the novelty of our paper is to use the concept of the closed loop supply chain coupled with a mathematical model for modeling smart electrical grid systems.

This paper is organized as follow: the next section presents a literature review about smart grid

\footnotetext{
* Manuscript received June 12, 2014; revised July 9, 2014.

Corresponding author: Nathalie Saker; Tel.: +0097444547399; E-mail address: nsaker@qf.org.qa.

doi: $10.12720 /$ sgce.4.1.8-14
} 
architectures as well as appropriate models. The third section presents a proposed model of smart grids based on concepts of closed loop supply chain. Then, the problem is formulated as a mathematical optimization problem whose objective is to minimize the costs of the whole system. In the fourth section, an illustrative example is used to show the ease and the robustness of the methodology. Finally, conclusions and suggested extensions are presented in the fifth section.

\section{Brief Literature on Smart Grid Architectures and Their Analytical Models}

The smart grid results from the implementation of various enabling power system automation, communication protection and control technologies that allow real time interoperability between end users and energy providers in order to enhance efficiency in utilization decision making [4].

\subsection{Smart grid architectures}

Many architectures of smart grids are presented in the literature like [1]-[7]. In [1], a network architecture is presented for smart grids that consist of renewable energy resources, synchrophasors, smart meters, sensors (to detect faults), etc. In [2], a two way communication network system is presented which consists of a macro grid, that is a traditional centralized power grid, and a micro grid that represents a decentralized community-scale power grid. In [4] an illustrative macro smart grid is presented but it was not detailed since it was developed for educational purposes.

\subsection{Smart grid models}

The objective of this subsection is to present the main mathematical models applied to smart grid architectures. Often, they are formulated as optimization problems; the objective of each one of these problems is to determine the power flows values within lines while minimizing the total costs of the system. For instance, the paper presented in [6] consists of modeling the resource allocations, the power flows and the load control technologies in order to find the optimal combination and the optimal architecture of the smart grid for given electricity demand, renewable energies are considered but the optimisation method is not clearly detailed. In [7], the optimization potential of domestic technologies (e.g. DR actions applied on electrical loads) is exploited that allows prosumers to participate in the electricity production process. In [10], a linear programming model is developed to identify the optimal combination of supply sources, the demand sites to serve, and the pathways along which the reallocated power should flow. This model takes into account the uncertainties associated with the alternative sources as wind power but it does not take into account the DR actions. Reference [5] presented a home energy management system that consists of an optimization model of demand side management considering the customer behavior as well as the presence of distributed generation (e.g. PVs and wind turbines) and storage facilities (e.g. batteries, fuel cells and plug in hybrid electrical cars). This model is designed for home use but it was not extend to the power grid level. The proposed mathematical model in this paper is based on a classical approach of closed loop supply chain; it is presented in the next section and it is based on the model presented in [11].

For our paper, the most relevant reference is paper [3] as it proposes to represent a smart grid architecture as a closed loop supply chain. However, this paper did not address the modeling and optimization issues of smart grid systems. This is precisely the gap we propose to develop in our paper.

\section{Modeling Smart Grid Using Closed Loop Supply Chain Concepts}

We present in the next two subsections the smart grid architecture inspired by paper [3], our modeling methodology as well as the resulting mathematical optimization problem.

\subsection{A closed loop supply chain representation of a smart grid architecture}

The smart grid architecture discussed in [3] is reproduced in Fig. 1. 


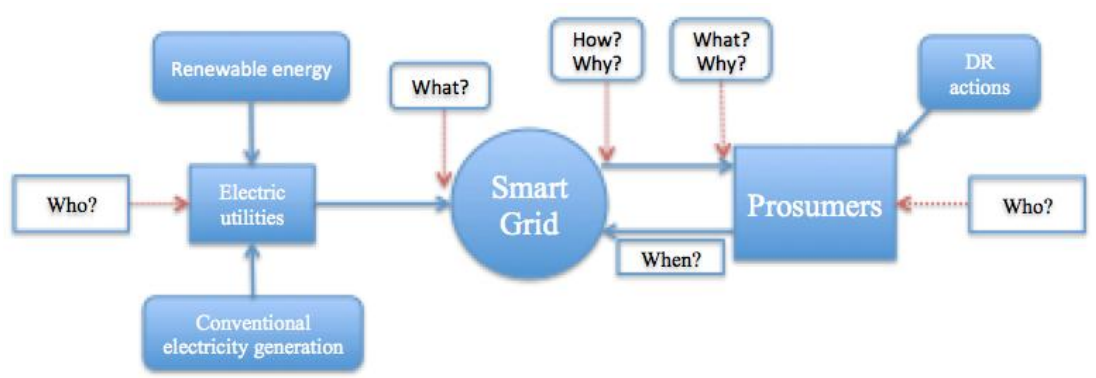

Fig. 1. A smart grid architecture using a concept of closed loop supply chain [3].

The architecture presented in Fig. 1 contains five basic components that are "Who", "How", "What", "Why" and "When". They are interrelated and their combination identifies to a large extent the types of issues arising from the resulting reverse logistics system. This model is much more developed than the one used in [8] because the first considers that supply must be equal to the demand and that represents an essential condition for keeping the power system stable.

Next, we detail the different elements of the model presented in Fig. 1. The electric utility is an electric power company that engages in the generation, transmission and distribution of electricity for sale generally in an electrical market. The prosumers represent active consumers that consume and control their electrical power consumption. The controlled electrical power is a load reduction resulted from applying Demand Response (or DR) actions; DR actions refer to direct curtailment actions implemented at the customer side in response to particular conditions within the power system (such as peak periods and outages) [9]. As seen in Fig. 1, "Who" means who is involved, the response is electric utilities, the Operator of the power system and the prosumers (representing several hundred of thousands of households). "What" means what is fed into the grid, the response is the electrical power produced from: (i) Conventional power generation, (ii) from small and large scale renewable energy generation units (e.g. wind farms or solar panels) and (iii) from prosumers as a form of small scale controlled power (several $\mathrm{kWs}$ only) through the application of DR actions. "How" means how the power provision is processed, the response is that the transmission lines take a primordial role in linking between the central and the huge generation units; the distribution lines take the role of linking between the prosumers and the smart grids. "Why" means why the reverse flows are initiated and accepted, the response is that the reverse flows are initiated by consumers starting to control their consumption to be returned back to the grid, they are remunerated by the power system operator in function of the curtailed power. "When" means when the loop is closed, the response is when balancing between electricity supply and demand is needed. In supply chain management, reverse logistics is a broad term referring to the logistic processes that include reverse distribution of goods and information to flow in the opposite direction of normal logistics activities [8]. For power system management, the reverse logistics could be represented by the reverse power flows generated from prosumers with DR actions applied on their electrical loads since the power flows in this case from consumers to producers. The aggregator is the mediator between the prosumers and the electrical market; it collects the power flows from many prosumers in order to sell it back to the electric power system (or the electric utilities). Modeling the smart grid (see Fig. 1) using an approach of closed loop supply chain permits: (i) a better comprehension of its architecture since it presents clearly the power flows directions, and (ii) managing the different centralized generation units and the multi directional power flows in the electrical grid; but, presenting an architecture of the smart grid should be associated with a mathematical model as presented next.

\subsection{Mathematical model}

The objective here is to present the main mathematical models applied to smart grid architectures. Often, they are formulated as optimization problems whose objective is to determine the optimal architecture and the power flows within the lines that minimize the total costs of the system. This is precisely what we propose to develop in this section. The system presented in Fig. 1 can be modeled as 
depicted in Fig. 2. As shown, Fig. 2 is partly a closed loop network. As in [11], this problem is treated as a classical closed loop supply chain optimization problem.

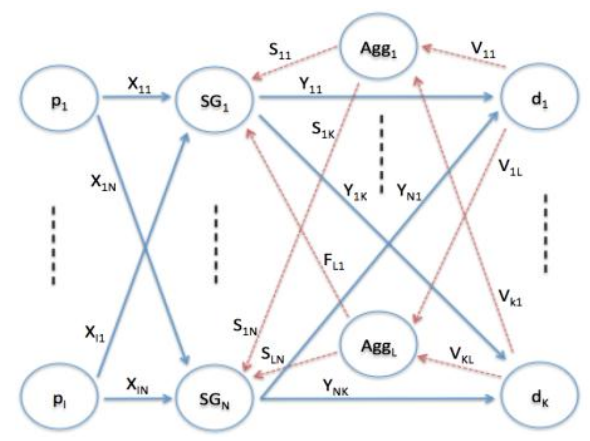

Fig. 2. Smart grid architecture model.

In order to develop the mathematical optimization model presented in Fig. 2, it is necessary to define the abbreviations, the group of sets, the parameters, the decision variables, the objective function as well as the constraints.

\section{Abbreviations:}

Aggregator: $A g g_{l}$

Prosumers: $d_{k}$

Smart distribution grid (or the electricity supplier): $S G_{n}$

Plant: $p_{i}$

Sets:

I: set of plants $(1,2, \cdots, i, \cdots, I)$.

$N$ : set of smart distribution grids $(1,2, \cdots, n, \cdots, N)$.

$K$ : set of prosumers $(1,2, \cdots, k, \cdots, K)$.

$L$ : set of aggregators $(1,2, \cdots, l, \cdots, L)$.

\section{Parameters:}

$A_{i}$ : Production costs of plant $p_{i}$ per MW.

$B_{i}$ : Electricity transmission cost between plant $p_{i}$ and $S G_{n}$ (it depends on the voltage level) per MW.

$C_{n}$ : Distribution cost between $S G_{n}$ and the prosumers per MW, it depends on the contract of electricity supply signed between the electric utilities and the prosumers.

$D_{k}$ : Controlling costs (via DR actions) in the demand side per MW.

$E_{k l}$ : Power reduction cost per MW of prosumer $d_{k}$ contracted with the aggregator $A g g_{l}$.

$F_{l n}$ : Cost of aggregated power per MW collected by $A g g_{l}$ and delivered to $S G_{n}$.

$G_{i}$ : Fixed installation costs of the plant $p_{i}$ per MW.

$H_{l}$ : Fixed operating costs of the aggregator $\mathrm{Agg}_{l}$.

$P_{i}$ : Capacity of the power plant $p_{i}$ in MW.

$R_{n}$ : Capacity of the smart grid $S G_{n}$ in MW.

$Q_{l}$ : Capacity of the aggregator $A g g_{l}$ in MW.

dem $_{k}$ : Effective consumption of prosumer $d_{k}$ in MW.

dem_Agg: Demand of $A g g_{l}$ in MW.

Decision Variables:

$X_{i n}$ : Power flow value from plant $p_{i}$ to $S G_{n}$ measured in MW.

$Y_{n k}$ : Power flow value from $S G_{n}$ to the prosumer $d_{k}$ measured in MW.

$V_{k l}$ : Value of reduced (or controlled) electrical power (via DR actions applications) delivered from prosumer $d_{k}$ to $A g g_{l}$ and measured in MW.

$S_{l n}$ : Value of aggregated power delivered from $\mathrm{Agg}_{l}$ to $S G_{n}$ measured in MW.

$Z_{i}=1$, if a plant $p_{i}$ is operating, 0 , otherwise.

The objective function of the optimization problem is to minimize the total costs of the system (see equation (1)). 


$$
\operatorname{Min}\left\{\sum_{i} G_{i} P_{i} Z_{i}+\sum_{l} H_{l}+\sum_{i} \sum_{n}\left(A_{i}+B_{i}\right) X_{i n}+\sum_{n} \sum_{k} C_{n} Y_{n k}+\sum_{k} \sum_{l}\left(D_{k}+E_{k l}\right) V_{k l}+\sum_{l} \sum_{n} F_{\mathrm{ln}} S_{l n}\right\}
$$

The first two parts of the objective function represent respectively the installation costs of the plants, and the fixed operation costs of the aggregators; The third and the fourth part represent respectively (i) the production and transmission costs of electricity and (ii) the electricity distribution costs, the fifth part is related to: (i) the controlling costs (via DR actions) and (ii) load reduction costs paid by the aggregators to prosumers for the controlled power. The final part is related to the costs of aggregated power delivered from the aggregator participating in the electricity market to the smart distribution grids. The constraints of the mathematical model are presented from (2) to (12).

$$
\begin{aligned}
& \sum_{n} X_{i n} \leq P_{i} \cdot Z_{i} \quad \forall i \\
& \sum_{i} X_{i n}+\sum_{l} S_{\mathrm{ln}} \leq R_{n} \quad \forall n \\
& \sum_{k} V_{k l}=\sum_{n} S_{\mathrm{ln}} \quad \forall l \\
& \sum_{l} S_{\mathrm{ln}}+\sum_{i} X_{i n}=\sum_{k} Y_{n k} \quad \forall n \\
& \sum_{n} Y_{n k} \geq d e m_{k} \quad \forall k \\
& \sum_{n} Y_{n k} \geq \sum_{l} V_{k l} \quad \forall k \quad \forall \\
& \sum_{n} Y_{n k}=d e m_{k}+\sum_{l} V_{k l} \quad \forall k \\
& \sum_{k} V_{k l} \leq Q_{l} \quad \forall l \\
& \sum_{k} V_{k l}=d e m_{-} A g g_{l} \quad \forall l \\
& Z_{i} \in\{0,1\} \quad \forall i \quad \\
& X_{i n}, Y_{n k}, V_{k l}, S_{\mathrm{ln}} \geq 0 \quad \forall i, n, k, l
\end{aligned}
$$

Equation (2) ensures that the delivered electricity to each smart distribution grid from plant $p_{i}$ is inferior or equal to the capacity of this power plant, (3) ensures that the sum of the incoming flows from the power plants and from the aggregators to each smart distribution grid is inferior or equal to its capacity, (4) ensures that the sum of the incoming flows of an aggregator is equal to the sum of its outgoing flows, (5) ensures that the incoming flows of each smart distribution grid is equal to the sum of its outgoing flows, (6) ensures that the demand of each prosumer is satisfied, (7) ensures that the sum of the direct flows is greater than the sum of the reverse flows, (8) is the balancing equation of incoming and outcoming flows for each prosumer, (9) is the capacity constraints of each aggregator, (10) ensures that the demand of each aggregator is satisfied, (11) represents the binary nature of the decision variable $Z_{i}$ and finally (12) preserves the non-negativity restriction of the decision variables. The resulting model is a mixed integer nonlinear programming problem which can be resolved, depending on the complexity of the relationships, using MATLAB or software packages such as Cplex or other similar products.

\section{Numerical Results of a Case Study}

In the next two subsections, we develop an illustrative example and we solve the problem to show the ease and robustness of the approach.

\subsection{Presentation of the case study}

A case study is built where sets and parameters values were given, an example of the French power sector is used in order to present the different smart grid elements (see Fig. 2) ; this grid consists of the 
following parts. (i) Three power plants, $p_{a}, p_{b}$ and $p_{c}$ : they are located in France, the first one presents the gas power plant of Montoire de Bretagne with a capacity of $435 \mathrm{MW}$, the second one is the wind turbine generation plant of Eure et Loire with a capacity of $52 \mathrm{MW}$ and the third one is the gas power plant of Fos-sur-mer with a capacity of $480 \mathrm{MW}$; the construction cost per MW, $G_{i}$, is equal to $442000 €$ for the gas power plants, and to 1,3 million $€$ for the wind turbine; their production costs per $\mathrm{MW}, A_{i}$, are equal to $27 €$ for the gas power plants, and to $82 €$ for the wind turbine; [12]-[15]. (ii) Two smart distribution grids, $S G_{a}$ and $S G_{b}$ : the capacity, $R_{n}$, of each one is equal to $3000 \mathrm{MW}$. The transmission cost of electricity used in the model depends on the voltage level, for $H T B$ voltage level (that corresponds to transmission voltage values higher than $50 \mathrm{kV}$ ), the transmission cost, $B_{i}$, is about $40 €$ per $\mathrm{MW}$, and for $H T A$ voltage level (that corresponds to voltage levels lower than $50 \mathrm{kV}$ ), the transmission cost is about $30 €$ per MW, [16]. The distribution costs of electricity, $C_{n}$, depend on the subscribed electricity supply option by prosumers; for example, for a basic supply option, the cost of electricity consumption is about 137,2 $€$ per MW, and for an Off-Peak supply option, the cost is equal to $105 €$ per MW [17]. (iii) Two aggregators, $A g g_{a}$ and $A g g_{b}$ and two prosumers, $d_{a}$ and $d_{b}$ : DR action control costs, $D_{k}$, for each prosumer are taken equal to $50 €$ per MW and the controlled power of prosumers is sold to the aggregators for a price, $E_{k l}$, approximatively equals to $50 €$ per MW [18], $F_{l n}$ values are taken around $60 €$ per MW (higher than $50 €$ for considering aggregator profits). The effective consumption, $d_{e m}$ of each prosumers, $d_{a}$ and $d_{b}$ is taken equal to $400 \mathrm{MW}$. The operation cost, $\mathrm{H}$, of each aggregator, $A g g_{a}$ and $A g g_{b}$ is respectively equal to 5000 $€$ and $15000 €$, and the demand of each, dem_Aggl, is taken respectively equal to 200 and $300 \mathrm{MW}$; finally, the capacity of each aggregator $Q_{l}$ is supposed equal to $1000 \mathrm{MW}$. The GNU Linear Programming Kit (GLPK) is used for solving the optimization problem presented in the subsection 3.2. GLPK is a software package intended for solving large-scale Linear Programming (LP), Mixed Integer Programming (MIP), and other related problems [19].

Table 1. Optimization results using GLPK

\begin{tabular}{cccc}
\hline \hline Variable name & Optimized value (MW) & Variable name & Optimized value (MW) \\
\hline $\mathrm{X}\left[\mathrm{p}_{\mathrm{a}}, \mathrm{SG}_{\mathrm{a}}\right]$ & 0 & $\mathrm{~V}\left[\mathrm{ka}, \mathrm{Agg}_{\mathrm{a}}\right]$ & 200 \\
$\mathrm{X}\left[\mathrm{p}_{\mathrm{p}}, \mathrm{SG}_{\mathrm{b}}\right]$ & 320 & $\mathrm{~V}\left[\mathrm{ka}, \mathrm{Agg}_{\mathrm{b}}\right]$ & 300 \\
$\mathrm{X}\left[\mathrm{p}_{\mathrm{b}}, \mathrm{SG}_{\mathrm{a}}\right]$ & 0 & $\mathrm{~V}\left[\mathrm{~kb}, \mathrm{Agg}_{\mathrm{a}}\right]$ & 0 \\
$\mathrm{X}\left[\mathrm{p}_{\mathrm{b}}, \mathrm{SG}_{\mathrm{b}}\right]$ & 0 & $\mathrm{~V}\left[\mathrm{~kb}, \mathrm{Agg}_{\mathrm{b}}\right]$ & 0 \\
$\mathrm{X}\left[\mathrm{p}_{\mathrm{c}}, \mathrm{SG}_{\mathrm{a}}\right]$ & 0 & $\mathrm{~S}\left[\mathrm{Agg}_{\mathrm{a}}, \mathrm{SG}_{\mathrm{a}}\right]$ & 0 \\
$\mathrm{X}\left[\mathrm{p}_{\mathrm{c}}, \mathrm{SG}_{\mathrm{b}}\right]$ & $\mathrm{S}\left[\mathrm{Agg}_{\mathrm{a}}, \mathrm{SG}_{\mathrm{b}}\right]$ & 200 \\
$\mathrm{Y}\left[\mathrm{SG}_{\mathrm{a}}, \mathrm{k}_{\mathrm{a}}\right]$ & 080 & $\mathrm{~S}\left[\mathrm{Agg}_{\mathrm{b}}, \mathrm{SG}_{\mathrm{a}}\right]$ & 0 \\
$\mathrm{Y}\left[\mathrm{SG}_{\mathrm{a}}, \mathrm{k}_{\mathrm{b}}\right]$ & 0 & $\mathrm{~S}\left[\mathrm{Agg}_{\mathrm{b}}, \mathrm{SG}_{\mathrm{b}}\right]$ & 300 \\
$\mathrm{Y}\left[\mathrm{SG}_{\mathrm{b}}, \mathrm{k}_{\mathrm{a}}\right]$ & & $\mathrm{Z}\left[\mathrm{p}_{\mathrm{a}}\right]$ & 1 \\
$\mathrm{Y}\left[\mathrm{SG}_{\mathrm{b}}, \mathrm{k}_{\mathrm{b}}\right]$ & 900 & $\mathrm{Z}\left[\mathrm{p}_{\mathrm{b}}\right]$ & 0 \\
& 40 & $\mathrm{Z}\left[\mathrm{p}_{\mathrm{c}}\right]$ & 1 \\
\hline \hline
\end{tabular}

\subsection{Results: Optimal solutions}

The optimal solutions are presented in this subsection (see Table 1); they consist of power flow values in the different branches of the smart grid architecture and the binary variables describing which power plants should be open and which ones should be closed in order to minimize the system total costs. The optimal total cost of the whole system is equal to $411290100 €$ and the optimal power flow values between the plants, the smart distribution grids, the prosumers, and the aggregators are shown in Table 1.

In the optimal solution, the gas power plants $p_{a}$ and $p_{c}$ should be open and operating while the wind turbine generation unit, $p_{b}$ should be closed.

\section{Conclusions and Further Developments}

A brief literature review about smart grid architecture models and the associated mathematical models is presented in this paper. A gap in the literature is identified and a novel approach, using concepts from closed loop supply chain, is developed in this paper. The architecture is modeled and formulated as a mathematical optimization problem with an objective function to minimize the total costs of the system. An illustrative simple model is used to show the robustness of the proposed approach. This paper can be 
extended to take into account other sources of energies (e.g. renewable energies integration in the demand side (prosumers side)); the model will be extended to take into account additional binary variables associated with the aggregators and the smart distribution grids. A large-scale model will be developed to handle an application relative to a national grid. Other factors will also be considered such as the uncertainties relative to the demand as well as the unreliability of some smart grid components.

\section{References}

[1] Radhika N, Sivalingam K, Anand V. Network architecture for smart grids. In: Proc. International Conference on Computer, Communication and Electrical Technology, 2011: 24-26.

[2] Kim JY, Choi HK. An efficient versatile key management protocol for secure smart grid communications. In: Proc. IEEE Wireless Communications and Networking Conference: Mobile and Wireless Networks, 2012: 1823-1828.

[3] Richter A, Laan E, Ketter W, Valogianni K. Transitioning from the traditional to the smart grid: Lessons learned from closedloop supply chains. In: Proc. International Conference on Smart Grid Technology, Economics and Policy, 2012:1-7.

[4] Reed G.F. Smart grid education models for modern electric power system engineering curriculum. In: Proc. IEEE Power and Energy Society General Meeting, 2010:1-5.

[5] El-Tolba E.H, Maaroufi M, Ouassaid M. Demand side management algorithms and modeling in smart grids: A customer's behavior based study. In: Proc. International Renewable and Sustainable Energy Conference, 2013:531-536.

[6] Das A, Balakrishnan V. Energy service companies (ESCOs) to optimize power in peak demand period in hybrid energy system: An impact on climate change. In: Proc. IEEE Green Technologies Conference, 2010:1-10.

[7] Molderink A, Bakker V, Bosman M.G.C, Hurink J.L, Smit G.J.M. Management and control of domestic smart grid technology. IEEE Transactions on Smart Grid, 2010; 1(2):109-119.

[8] De-Brito M, Dekker R. A framework for reverse logistics. In: Dekker R, Fleishmann M, Inderfurth K and Van Wassenhove L.N, editors: Reverse Logistics. Quantitative models for Cloosed-Loop Supply Chains, Berlin; 2003:1-25.

[9] J.Torriti, M.G.Hassan, M.Leach. Demand response experience in Europe: Policies, programmes and implementation, Energy Journal, April 2010:1575-1583.

[10] Nygard K.E, Ghosn S.B, Chowdhury M.M, Loegering D, McCulloch R, Ranganathan P. Optmization models for energy reallocation in a smart grid. In: Proc. IEEE Conference on Computer Communications Workshops (INFOCOM WKSHPS), 2011:186-190.

[11] Hassanzadeh A. Design and optimization of closed-loop supply chain management. PhD dissertation. Department of Industrial and Manufacturing Systems Engineering, University of Winsdor. Winsdor, Ontario, Canada; 2012.

[12] Global Energy Observatory. (June 2014). List of Gas Power plants. [Online]. Available: http://globalenergyobservatory.org/list.php?db=PowerPlants\&type=Gas

[13] U.S. Energy Information Administration (iea). (December 2013). Average Power Plant Operating Expenses. [Online]. Available: http://www.eia.gov/electricity/annual/html/epa_08_04.html

[14] U.S. Energy Information Administration (iea). (December 2013). Projected Costs of Generating Electricity. [Online]. Available: http://www.iea.org/textbase/npsum/eleccostsum.pdf

[15] Windustry. (May 2014). How much do wind turbine cost? [Online]. Available: http://www.windustry.org/ resources/howmuch-do-wind-turbines-cost

[16] RTE. (Aout 2010). Comprendre le Tarif. [Online]. Available: http://clients.rte-France.com/htm/fr/mediatheque/ telecharge/ comprendre_le_tarif_01_08_2010.pdf

[17] EDF. (2012). Fournisseurs d'electricite. [Online]. Available: http://www.fournisseurs-electricite.com/edf-electricitereglemente-particuliers.

[18] F.M.Andersen. (October 2006). Analyses of demand response in Denmark. [Online]. Available: http://orbit.dtu.dk/fedora/ objects/orbit:88362/datastreams/file_7703292/content

[19] Wikipedia. (March 2014). GNU Linear Programming Kit. [Online]. Available: http://en.wikipedia.org/wiki/ GNU_Linear_Programming_Kit 